\title{
PP012 - Avaliação do efeito antiparasitário do omeprazol na prevenção do desenvolvimento de lesões cutâneas em hamsters infectados por Leishmania brasiliensis Evaluation of omeprazole's antiparasitary effect preventing the development of cutaneous lesions due to Leishmania brasiliensis in hamsters"
}

Hélio Amante Miot ${ }^{1}$

Ana Laura Bastos da Costa ${ }^{3}$
Luciane Donida Bartoli Miot ${ }^{2}$ Cristiane Yuri Matsuo $^{4} \quad$ Lúcia Helena O'Dwyer ${ }^{5}$

Resumo: Fundamentos - A leishmaniose tegumentar americana permanece doença endêmica em diversas regiões brasileiras. A sobrevivência do parasita no interior dos macrófagos se deve, em parte, pela atividade de uma $\mathrm{K}^{+} / \mathrm{H}^{+}$-ATPase de membrana que pode ser inibida pelo omeprazol.

OBJETIVOS - Avaliar a eficácia do omeprazol na prevenção do desenvolvimento de lesões de leishmaniose em hamsters. MÉToDos - Empregaram-se 18 hamsters, divididos em três grupos: o grupo L recebeu apenas a inoculação de L. brasiliensis na pata anterior direita, o grupo $\mathrm{O}$ recebeu apenas doses diárias de $0,4 \mathrm{mg}$ de omeprazol subcutâneo, e o grupo $\mathrm{L}+\mathrm{O}$ recebeu o inóculo de leishmanias e o tratamento com omeprazol desde o dia da inoculação. $\mathrm{O}$ estudo foi conduzido por 42 dias, realizaram-se medidas dos diâmetros das patas semanalmente, $\mathrm{e}$, ao final do estudo, foram realizados esfregaços das lesões para verificação dos parasitas.

REsultados - Os hamsters dos grupos L e L+O desenvolveram lesões de leishmaniose tegumentar havendo ulceração em duas patas do grupo L e uma do grupo L+O. Ao final do estudo, a mobilidade e vitalidade no grupo $\mathrm{L}$ foram menores que em $\mathrm{L}+\mathrm{O}$, e estas menores que no grupo $\mathrm{O}$. Os diâmetros das patas inoculadas nos grupos L e L+O foram significativamente maiores que no início do estudo $(\mathrm{p}<0.05)$. Não houve diferença significativa entre os diâmetros das patas dos grupos L e $\mathrm{L}+\mathrm{O}$ ao final do estudo $(\mathrm{p}>0,05)$, sendo detectados parasitas no esfregaço das lesões dos dois grupos.

CONCLusões - Omeprazol, no protocolo utilizado, não evitou o desenvolvimento de lesões de leishmaniose tegumentar em hamsters.

Palavras-chave: Leishmania braziliensis; Leishmaniose; Omeprazol

\begin{abstract}
BACKGROUND - Cutaneous leishmaniasis remains an endemic disease in several brazilian regions. The parasite' survival in macrophages is due to a membrane $K^{+} / H^{+}$-ATPase that can be inbibited by omeprazole. OBJETVES - Evaluate omeprazole's efficacy preventing the development of cutaneous leishmaniasis in hamsters. METHODS - Eighteen hamsters were divided in 3 groups: the L group received an inoculation of L. brasiliensis on right paw, the $O$ group received daily 0,4mg omeprazole subcutaneously, and $L+O$ group received both omeprazole and the inocule. The study was performed in 42 days, and the measurements of the diameter of paws were done weekly. At the end of the study was carried out a smear to verify the presence of parasites. RESULTS - Hamsters fitted in $L$ and $L+O$ groups have developed cutaneous leishmaniasis' lesions, there were ulcerations in 2 hamsters from $L$ group and 1 from $L+O$ group. At the end of the study, the vitality and mobility of animals from $L$ group were less prominent than $L+O$ and $O$ groups. Diameters of inoculated paws in $L$ and $L+O$ groups were significantly larger in comparison to begin of the study $(p<0,05)$. There were no statistical difference between diameters of the paws from $L$ and $L+O$ groups at the end of study $(p>0,05)$. Parasites were detected at microscopic smears of lesions from both groups.

ConClusions - Hamsters' cutaneous lesions due to Leishmania sp. inoculation couldn't be prevented by omeprazole, using this protocol.

Keywords: Leishmania brasiliensis; Leishmaniasis; Omeprazole
\end{abstract}

\footnotetext{
* Trabalho realizado no Departamentos de Dermatologia e Radioterapia - FMB - Unesp, Botucatu (SP), Brasil - Departamento de Parasitologia - IBB - Unesp, Botucatu, São Paulo (SP), Brasil

Professor doutor substituto do Departamento de Dermatologia da Unesp - Botucatu (SP), Brasil.

Pós-graduanda (doutorado), Dermatologista do Departamento de Dermatologia da Unesp - Botucatu (SP), Brasil.

Acadêmica de medicina da Faculdade de Medicina da Unesp - Botucatu (SP), Brasil.

Acadêmica de medicina da Faculdade de Medicina da Unesp - Botucatu (SP), Brasil

Professora-assistente doutora do Departamento de Parasitologia da Unesp - Botucatu (SP), Brasil 


\section{INTRODUÇÃO}

A leishmaniose tegumentar americana permanece doença endêmica em diversas regióes brasileiras, causando grande morbidade e custos para a saúde pública. ${ }^{1}$

Seu agente etiológico, Leishmania brasiliensis, é um protozoário dimórfico que existe como promastigota flagelado no intestino de mosquitos e como amastigota, sem flagelo, no interior de macrófagos de mamíferos.

O ambiente dos fagolisossomas macrofágicos é ácido em ratos, hamsters, seres humanos e nos camundongos em virtude da riqueza de prótons disponíveis nesse meio. As formas amastigotas do protozoário realizam atividades como respiração, percepção de nutrientes e síntese de RNA e DNA de maneira eficaz em $\mathrm{pH}$ de 5,0 até 5,5. A percepção de nutrientes ocorre por um mecanismo de transporte de prótons que leva a um acúmulo desse produto no interior dos parasitas. Esse efeito é controlado por uma bomba $\mathrm{K}^{+} / \mathrm{H}^{+}$-ATPase tipo P situada na superfície da membrana do protozoário, a qual desloca os prótons de seu citoplasma para o interior dos lisossomos. Isso permite que os parasitas mantenham $\mathrm{pH}$ interno neutro e estável dentro dos fagolisossomos ricos em prótons. ${ }^{2}$

Em virtude desse papel da bomba $\mathrm{K}^{+} / \mathrm{H}^{+}$ATPase na manutenção do $\mathrm{pH}$ dos parasitas, esse sistema tornou-se um atrativo alvo para pesquisa de novas quimioterapias.

Os medicamentos disponíveis atualmente para o tratamento da leishmaniose apresentam efeitos colaterais, potencial toxicidade e vias de administração que reduzem a aderência ao tratamento, e ampla cobertura em saúde coletiva. ${ }^{3}$

O omeprazol é medicamento empregado no tratamento da úlcera péptica e não apresenta efeitos tóxicos relevantes, além da possibilidade de administração oral. Representa um importante inibidor da bomba $\mathrm{K}+/ \mathrm{H}+$-ATPase gástrica humana.

Em pH neutro, o omeprazol penetra as membranas celulares e se acumula em compartimentos celulares ácidos, como os lisossomas, onde se submete à protonação. A forma protonada torna-se um ativo composto (sulfonamida) e age como um potente inibidor dessa ATPase, fazendo com que os prótons fiquem acumulados no interior da célula. Na mucosa do estômago humano, o omeprazol ativado, ao inibir a bomba $\mathrm{K}^{+} / \mathrm{H}^{+}$-ATPase, interrompe a secreção ácida pelas células parietais. ${ }^{4}$

A bomba $\mathrm{K}^{+} / \mathrm{H}^{+}$-ATPase tipo $\mathrm{P}$ da superfície da membrana da Leishmania revelou-se um potencial alvo para o omeprazol, e sua inibição in vitro resultou em importante efeito leishmanicida. O acúmulo de omeprazol dentro dos lisossomas ácidos de macrófa- gos levou a considerar a hipótese de um possível efeito leishmanicida também in vivo. ${ }^{5}$

Neste estudo, avalia-se a atividade do omeprazol na prevenção de lesões em hamsters infectados pela Leishmania brasiliensis.

\section{MATERIAL E MÉTODOS}

Estudo experimental envolvendo 18 hamsters de mesma linhagem acondicionados em três criadouros e submetidos a igual regime alimentar e hídrico por 42 dias, em biotério climatizado. O grupo "O" recebeu diariamente injeções subcutâneas na região dorsal de $0,1 \mathrm{ml}$ de solução de omeprazol $40 \mathrm{mg} / \mathrm{ml}$ (Laboratório Cristália). O grupo "L" recebeu um inóculo de $0,1 \mathrm{ml}$ de solução contendo Leishmania brasiliensis na pata anterior direita no primeiro dia do estudo. A cepa foi proveniente de infecção canina oriunda da cidade de Conchas, São Paulo, cultivada em hamsters no Departamento de Parasitologia da Unesp de Botucatu. O grupo " $\mathrm{L}+\mathrm{O}$ " recebeu tanto a inoculação de parasitas no primeiro dia como as injeções diárias de omeprazol.

Foram medidos os diâmetros das patas dianteiras dos animais semanalmente usando um paquímetro milimetrado. Após 42 dias, os hamsters foram sacrificados com clorofórmio e realizados esfregaços das patas inoculadas e corados com Giemsa para verificação da presença de formas amastigotas nas lesões.

Os resultados foram tabulados em MS-Excel $2000^{\mathrm{TM}}$ e analisados utilizando o software Bioestat 2.0.

Para a análise comparativa da evolução temporal dos diâmetros das patas dentro dos grupos foi empregado o teste de Wilcoxon. Para a análise comparativa dos diâmetros das patas entre os grupos foram empregados os testes de Mann-Whitney e Kruskal-Wallis. Foi adotado como significativo $\mathrm{p}<0,05$.

\section{RESULTADOS}

Ao início do estudo, os 18 hamsters apresentavam-se com aspecto saudável, com mobilidade e vitalidade preservadas. Os diâmetros médios iniciais das patas dos hamsters não diferiram entre os três grupos ( $p>0,05$ Kruskal-Wallis), tampouco quando comparados com os diâmetros das patas dianteiras contralaterais ( $p>0,05$ Wilcoxon).

Na primeira semana que se sucedeu à inoculação, verificou-se discreto edema nas patas inoculadas dos hamsters pertencentes ao grupo L e $\mathrm{L}+\mathrm{O}$. Esse efeito mensurado já demonstrava diferença estatisticamente significativa em relação ao comportamento do grupo $O$ a partir da segunda semana $(p<0,05$ Kruskal-Wallis), ampliando sua margem até o final do experimento (Gráfico 1). As patas dianteiras contrala- 
GráfICo 1: Evolução do diâmetro médio das patas anteriores direitas dos hamsters dos três grupos

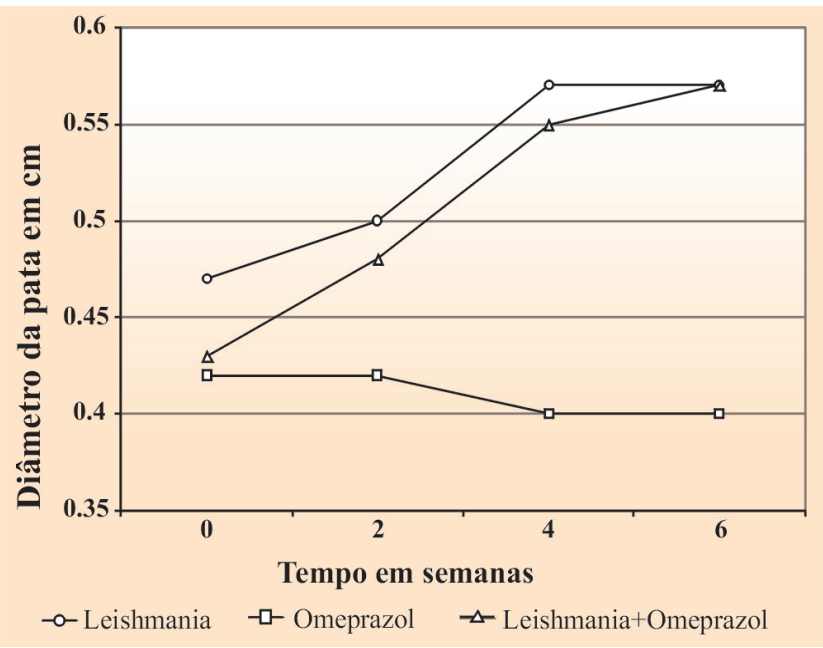

terais ao inóculo não apresentaram alteração em nenhum dos grupos.

Entre os animais infectados, houve franca ulceração nas patas de dois hamsters do grupo $\mathrm{L}$, a partir da quarta semana, e em um hamster do grupo $\mathrm{L}+\mathrm{O}$, a partir da quinta semana. Ao final do estudo, o eritema e o aspecto clínico infiltrativo das lesões do grupo $\mathrm{L}$ foram mais intensos que os do grupo $\mathrm{L}+\mathrm{O}$.

Todos os animais atingiram o final do estudo. Observou-se redução da mobilidade e vitalidade dos hamsters dos grupos L e L+O em comparação ao grupo $\mathrm{O}$, tendo a mobilidade do grupo $\mathrm{L}$ sido ainda menor que a do grupo $\mathrm{L}+\mathrm{O}$.

Após o término do estudo, os animais foram sacrificados, e o esfregaço das lesões das patas dos grupos $\mathrm{L}$ e $\mathrm{L}+\mathrm{O}$ apresentaram grande quantidade de amastigotas em todas as lâminas.

\section{DISCUSSÃO}

À medida que se compreendem as características moleculares dos mecanismos de defesa e sobrevivência de agentes infecciosos, abrem-se possibilidades de pesquisa para o desenvolvimento de agentes que interfiram em seu ciclo vital. ${ }^{2,7,8}$

A pesquisa de agentes leishmanicidas de baixa toxicidade, alta eficácia e comodidade posológica é um desafio que tem envolvido diversos grupos de pesquisa ao redor do mundo..$^{3,9-13}$

A redução de mais de $90 \%$ dos parasitas (Leishmania donovani) em culturas ácidas ou no interior de macrófagos in vitro empregando meios com diferentes concentrações de ommeprazol é um exemplo de interferência molecular direta no ciclo de vida do protozoário. ${ }^{2}$

Sendo o omeprazol um inibidor específico da bomba $\mathrm{K}^{+} / \mathrm{H}^{+}$- ATPase gástrica também em hamsters, seu efeito na bomba existente na superfície celular das leishmanias pôde ser avaliado nesses animais. ${ }^{14,15}$

Neste estudo preliminar, a escolha de altas doses de omeprazol relativas ao peso dos hamsters (120g) objetivou evitar falhas terapêuticas devido a baixos níveis terapêuticos da droga, e a escolha do início precoce do tratamento permitiu avaliar temporalmente o desenvolvimento das lesões nos diferentes grupos.

Entretanto, o uso subcutâneo do omeprazol, mesmo em doses elevadas desde a inoculação dos parasitas, não foi capaz de prevenir o desenvolvimento de lesões tegumentares de leishmaniose em hamsters, tampouco se observou marcante efeito leishmanicida ao exame microscópico.

As diferenças entre os aspectos clínicos das lesões inoculadas ao final do estudo, bem como a menor mobilidade dos hamsters, podem sugerir certo efeito antiinflamatório do omeprazol nos animais infectados, porém, para o tamanho da amostra utilizada, a possibilidade de esses achados representarem uma variabilidade individual deve ser considerada.

Outros protocolos de investigação, como o uso de inoculação em focinho de hamster (mais imunogênico), a injeção intralesional de omeprazol, uso de outras cepas de Leishmania sp., ou mesmo a associação com outras terapêuticas devem ser conduzidos para avaliar as possibilidades do emprego do omeprazol como agente antiparasitário contra a leishmaniose.

Ainda, outras drogas com ação semelhante ao omeprazol, como o lansoprazol, pantoprazol ou esomeprazol, podem desempenhar atividades leishmanicidas.

\section{CONCLUSÃO}

No protocolo utilizado, o emprego de omeprazol não preveniu o desenvolvimento de lesões de leishmaniose tegumentar em hamsters. 


\section{REFERÊNCIAS}

1. Ministério da Saúde. Fundação Nacional da Saúde. Manual de Controle da Leishmaniose Tegumentar Americana. 5 ${ }^{\text {a }}$ Ed. Brasília: FUNASA; 2000.

2. Jiang S, Anderson SA, Winget GD, Mukkada AJ. Plasma membrane $\mathrm{K}+/ \mathrm{H}+$-ATPase from Leishmania donovani. J Cell Physiol. 1994; 159:60-6.

3. Ercoli N, Coelho MV. Problems of drug evaluation in cutaneous leishmaniasis. Ann Trop Med Parasitol. 1967; 61:488-9.

4. Im WB, Sih JC, Blakeman DP, McGrath JP. Omeprazole, a specific inhibitor of gastric $\left(\mathrm{H}^{+}-\mathrm{K}^{+}\right)$-ATPase, is a $\mathrm{H}^{+}$activated oxidizing agent of sulfhydryl groups. $\mathrm{J}$ Biol Chem. 1985; 260:4591-7.

5. Jiang S, Meadows J, Anderson SA, Mukkada AJ. Antileishmanial activity of the antiulcer agent omeprazole. Antimicrob Agents Chemother. 2002; 46:2569-74.

6. Ayres M, Ayres Jr M, Ayres DL, dos Santos AS. Bioestat: 2.0 aplicações estatísticas nas áreas das ciências biológicas e médicas. Belém: Sociedade Civil Mamirauá; 2000.

7. Ogbunude PO, al-Jaser MH. Experimental chemotherapy of leishmaniasis with adenosine analogue Formycin A, in combination with inhibitor of nucleoside transport, nitrobenzylthioinosinate. Drugs Exp Clin Res. 1992; 18:423-6.

8. Rojas T, Avila JL. American Leishmania spp: formycin B treatment of cutaneous leishmaniasis in mice. Parasitology. 1987; 94:467-74.

9. Travi BL, Martinez JE, Zea A. Antimonial treatment of hamsters infected with Leishmania (Viannia) panamensis: assessment of parasitological cure with different therapeutic schedules. Trans R Soc Trop Med Hyg. 1993; 87:567-9.
10. Rasheid KA, Morsy TA. Efficacy of ivermectin on the infectivity of Leishmania major promastigotes. J Egypt Soc Parasitol. 1998; 28:207-12.

11. Travi B, Osorio Y. Failure of albendazole as an alternative treatment of cutaneous leishmaniasis in the hamster model. Mem Inst Oswaldo Cruz. 1998; 93:515.

12. Zeledon R, Soto R, Gonzalez G. Experimental superimposed infection of the hamster with Leishmania mexicana and L. braziliensis. Acta Trop. 1982; 39:367-72.

13. Brazil RP, Gilbert B. The action of oxamniquine on Leishmania braziliensis braziliensis in hamsters. Rev Inst Med Trop Sao Paulo. 1976; 18:87-8.

14. Chatterjee PK, Das PK. Characterization of Syrian hamster gastric mucosal $\mathrm{H}+, \mathrm{K}^{+}$-ATPase. Mol Cell Biochem. 1995; 148:95-103.

15. Kolbasa KP, Lancaster C, Olafsson AS, Gilbertson SK, Robert A. Indomethacin-induced gastric antral ulcers in hamsters. Gastroenterology. 1988; 95:932-44.

\footnotetext{
ENDEREÇO PARA CORRESPONDÊNCIA:

Hélio Amante Miot

Departamento de Dermatologia da FMB-UNESP

Campus universitário de Rubião Jr.

18618-000 - Botucatu - SP

Tel./Fax: (14) 3882-4922

E-mail: beliomiot@uol.com.br
} 